\title{
Posteromedial Subtalar Dislocation: A Case Report
}

Smail Abdouli ${ }^{12^{*}}$, Mannouk Rachid ${ }^{2}$, Hatim Abid $^{2}$, Mohammed Elidrissi $^{2}$, Abdelhalim Elibrahimi ${ }^{2}$, Abdelmajid Elmrini $^{2}$, Omar Kada ${ }^{1}$

${ }^{1}$ Orthopedic Department, Gonesse Hospital Center, 2 Boulevard du 19 Mars 1962, 95500 Gonesse, France

${ }^{2}$ Orthopedic Department, UHC HassanII, University Sidi Mohmmed Ben Abdellah, 3000 fez, Morocco

DOI: $10.36347 /$ sjams.2020.v08i05.027

| Received: 09.05.2020 | Accepted: 16.05.2020 | Published: 19.05.2020

*Corresponding author: Smail Abdouli

\section{Abstract}

\section{Case Report}

Subtalar dislocation is the simultaneous dislocation of the talocalcaneal and talonavicular joints of the foot, typically caused by falls from heights, twisting leg injuries and motor vehicle accidents. The vast majority, around $85 \%$, are medial dislocations whereas the lateral type occurs much less frequently. We report a medial subtalar dislocation, which was treated with closed reduction and cast for 6 weeks.

Keywords: Subtalar, dislocation, posteromedial, rare, closed reduction.

Copyright @ 2020: This is an open-access article distributed under the terms of the Creative Commons Attribution license which permits unrestricted use, distribution, and reproduction in any medium for non-commercial use (NonCommercial, or CC-BY-NC) provided the original author and source are credited.

\section{INTRODUCTION}

Subtalar dislocations are relatively uncommon injuries to the foot and ankle, accounting for $1 \%$ to $2 \%$ of all dislocations and $15 \%$ of peritalar injuries. However, they have the potential to cause substantial morbidity and functional disability [1]. Many of these dislocations result from high-energy injuries such as a fall from a height, athletic injuries, or a motor vehicle accident. Inversion or eversion force is dissipated through the weak talonavicular and talocalcaneal ligaments, which eventually results in subtalar dislocation. The head of the talus is found medially, and the rest of the foot is dislocated laterally in lateral subtalar dislocation; and the head of the talus is found laterally, and the rest of the foot medially in medial subtalar dislocation. Medial subtalar dislocations are encountered more frequently, with various studies reporting incidences varying between $65 \%$ and $85 \%$ [2]. Lateral dislocations are less common, found in 17 to $26 \%$ of cases, with rare case reports of both anterior and poster dislocations accounting for $1 \%$ of all subtalar dislocations [2,3]. We present a case of an adult with posteromedial subtalar dislocation after a fall while riding a motor cycle.

\section{CASE-REPORT}

A 23 year old male university student referred to our emergency service with a history of injury to his left ankle after a fall while riding a motor cycle. Clinical examination revealed a moderately swollen left covered by a skin abrasion with visible supination deformity of the forefoot and hindfoot [Figure1]. Dorsalis pedis and posterior tibial pulses were intact. Sensation in the foot was preserved and all flexor and extensor tendons were intact on initial examination. Radiographs of the ankle identified a posteromedial subtalar dislocation with medial displacement of the midfoot and hindfoot suggesting a subtalar dislocation [Figures 2(a) and 2(b)]. Under sedation, closed reduction was performed while the patient was under sedation, by placing the knee in 90 degrees of flexion and plantar-flexing the ankle, thereby relaxing the gastrocnemius-soleus complex. The hindfoot was then distracted and inverted, Postreduction radiographs demonstrated a well-reduced subtalar joint [Figures 3(a) and 3(b)], A computed tomography scan was obtained to delineate better the associated injuries and to assess for congruent reduction. CT showed a congruent reduction of the subtalar joint without evidence of any intraarticular debris [Figures 4(a) and 4(b)]. The patient was immobilized in a short leg cast for 6 weeks. A vigorous, active exercise program, progressive weightbearing, and active range-of-motion exercises to regain subtalar and midtarsal joint motion followed immobilization. After 40 months, the patient had a stable, relatively good functional foot, with minimal pain on walking on uneven ground. 


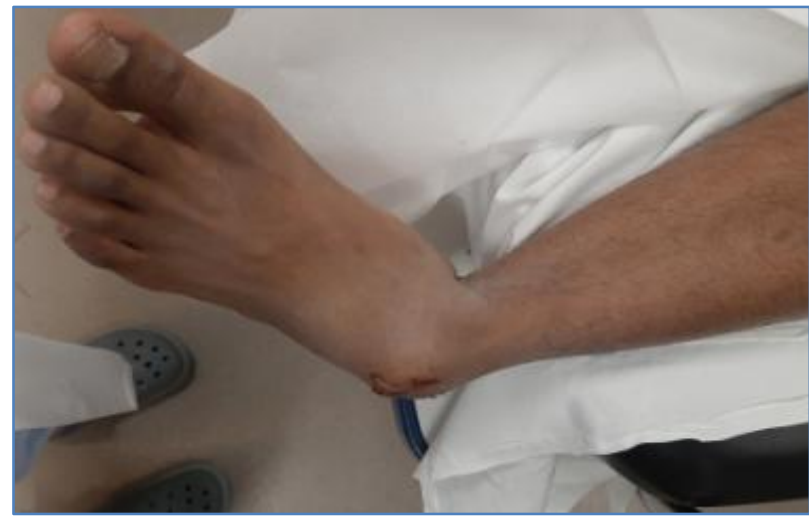

Fig-1: Physical exam findings for a medial subtalar dislocation.

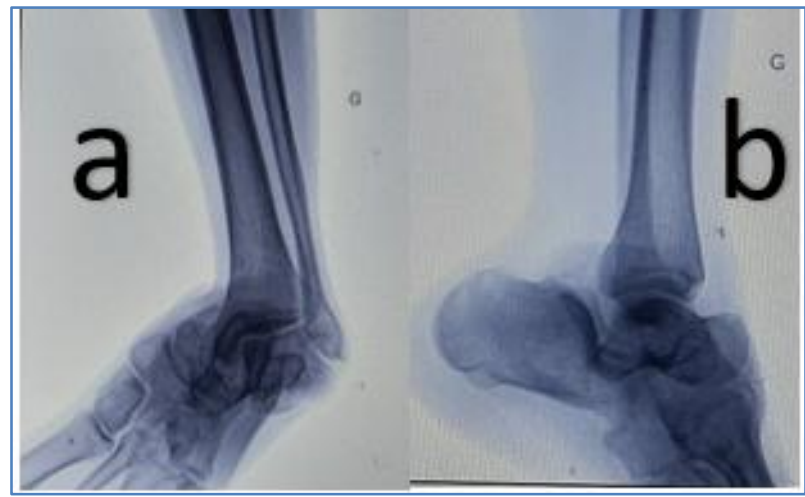

Fig-2: Injury radiographs demonstrating the posteromedial subtalar dislocation.

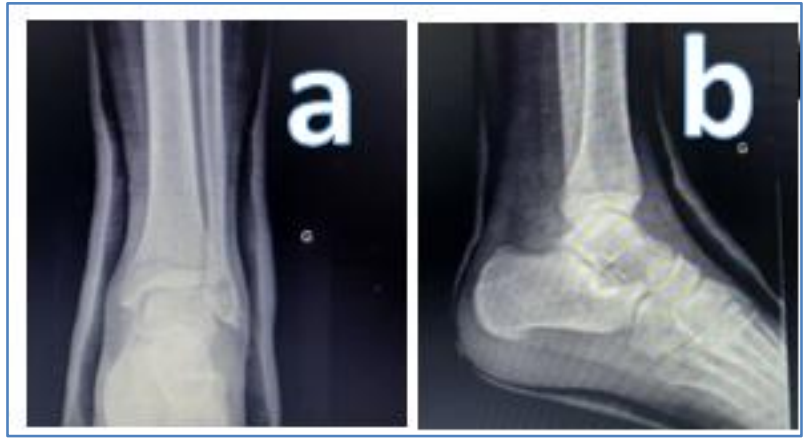

Fig-3: Postreduction radiographs demonstrating congruent of the subtalar joint.

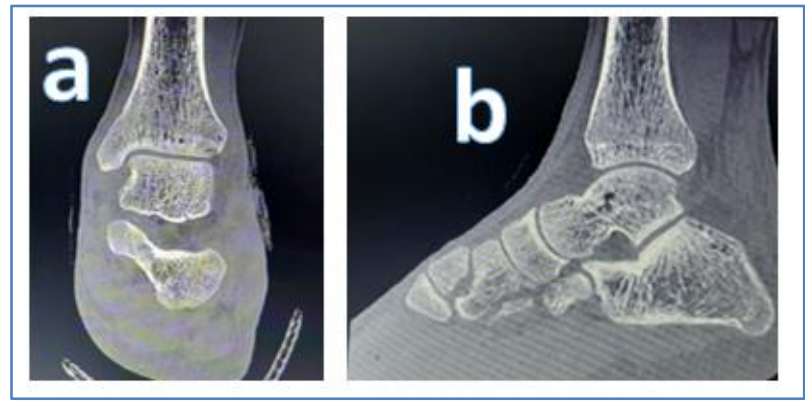

Fig-4: CT scan demonstrating congruent reduction of the subtalar joint without evidence of any intraarticular debris

\section{DISCUSSION}

Subtalar dislocation is an unusual orthopedic injury $[4,5]$. They constitute only $1 \%-2 \%$ of all dislocations [2]. The mechanism of injury involves simultaneous dislocation of the talocalcaneal and talonavicular joints and is most commonly the result of high-energy trauma [6]. Inversion of the foot results in a medial subtalar dislocation, whereas eversion produces a lateral dislocation. The strong calcaneonavicular ligament resists disruption, and the inversion or eversion force is dissipated through the weaker talonavicular and talocalcaneal ligaments, disrupting these 2 joints and allowing displacement of the calcaneus, navicular, and all distal bones of the foot as a unit, either medially or laterally $[7,8]$. The sustentaculum tali acts as a fulcrum about which the foot rotates to lever apart the talus and calcaneus in medial subtalar dislocation. The foot pivots about the anterior process of the calcaneus, again causing the talus and calcaneus to separate in lateral subtalar dislocation [9, 10] Rare cases of anterior [11] and posterior [9] displacement of the foot after subtalar dislocation have also been reported. It is important to distinguish the medial or lateral subtalar dislocations, because the method of reduction is different and the long-term prognosis appears to be worse with the lateral dislocation. Additionally, these injuries tend to be associated with fractures of the malleolus, talus, calcaneus, or fifth metatarsal [6]. The most important step in management is rapid reduction [4]. Reduction is performed with a flexed knee, traction and pressure opposite to the mechanism of injury [5]. Reduction should be closed when possible and followed by immediate immobilization in a splint. Post-reduction CT scans are recommended due to the high incidence of associated articular fractures which may require longer immobilization and be indicative of poorer prognosis $[4,12]$ In isolated dislocations, the leg should remain immobilized for one month followed by progressive weight-bearing and rangeof- motion exercises[4,6] Long-term outcomes after subtalar dislocation are affected by many factors including type of dislocation, severity of injury, associated fractures, and period of immobilization[13] Evidence suggests that lateral dislocations do worse than medial ones in the long-term, perhaps due to the greater complexity of anatomic damage[13] Some examples of late complications may include arthritis, avascular necrosis, and joint stiffness. Patients may experience pain, joint instability, and limp[14] According to most literature on the topic, $50 \%-80 \%$ of subtalar dislocation cases have post-reduction arthritis and decreased joint function[5] Open injuries have demonstrated high prevalence of post-traumatic osteoarthritis as well as osteonecrosis of the talus[15]Thus, lateral and open subtalar dislocations as well as those with associated fractures or neurovascular injury have shown to have a poorer prognosis than medial and closed dislocations. The best method to reduce the chances of developing such complications is immediate closed reduction, or open if necessary, under appropriate anesthesia. Reduction should be followed by a 4-6-week period of immobilization and then physical therapy [12]. 


\section{Conclusion}

Subtalar dislocations are rare injuries. They should be reduced as soon as possible to avoid soft tissue and circulatory complications. If closed reduction is unsuccessful, open reduction and stabilisation is indicated. Although early results may be reassuring, continued surveillance is necessary, as long-term outcomes may be affected by development of midfoot and hindfoot arthritis in closed subtalar dislocations.

\section{Conflicts of Interest}

None of the authors has any conflict of interest.

\section{REFERENCES}

1. Leitner B. Obstacles to reduction in subtalar dislocations. JBJS. 1954 Apr 1;36(2):299-306.

2. Bibbo C, Anderson RB, Davis WH. Injury characteristics and the clinical outcome of subtalar dislocations: a clinical and radiographic analysis of 25 cases. Foot \& ankle international. 2003 Feb;24(2):158-63.

3. Rammelt S, Goronzy J. Subtalar dislocations. Foot and ankle clinics. 2015 Jun 1;20(2):253-64.

4. Jerome JT, Varghese M, Sankaran B. Anteromedial subtalar dislocation. J Foot Ankle Surg. 2007;46(1):52-54.

5. Wagner R, Blattert TR, Weckbach A. Talar dislocations. Injury. 2004;35(2):36-45. 6. Giannoulis D, Papadopoulos DV, Lykissas MG,
KoulouvarisP, Gkiatas I, Mavrdontidis A. Subtalar dislocation without associated fractures: case report and review of literature. World $\mathrm{J}$ Orthop. 2015;6(3):374-379.

6. Grantham SA. Medial subtalar dislocation: five cases with a common etiology. Journal of Trauma and Acute Care Surgery. 1964 Nov 1;4(6):845-9.

7. Heppenstall RB, Farahvar H, Balderston R, Lotke P. Evaluation and management of subtalar dislocations. J Trauma. 20:494-497, 1980.

8. DeLee JD, Curtis R. Subtalar dislocation of the foot. J Bone Joint Surg. 64A:433-437,1982.

9. Monson ST, Ryan JR. Subtalar dislocation. J Bone Joint Surg. 63A: 1156-1158, 1981.

10. Inokuchi $\mathrm{S}$, Hashimoto $\mathrm{T}$, Usami $\mathrm{N}$. Anterior subtalar dislocation: case report. J Orthop Trauma. 11:235-237, 1997

11. Bohay DR, Manoli A. Subtalar joint dislocations. Foot Ankle Int. 1995;16(12):803-808.

12. Palma LD, Santucci A, Marinelli M, Borgogno E, Catalani A. Clinical outcome of closed isolated subtalar dislocations. Arch Orthop Trauma Surg. 2007;128(6):593-598.

13. Christensen SB, Lorentzen JE, Krogsøe O, Sneppen O. Subtalar dislocation. Acta Orthop Scand. 1977;48(6):707- 711.

14. Goldner JL, Poletti SC, Gates HS, Richardson WJ. Severe open subtalar dislocations. Long-term results. J Bone Joint Surg Am. 1995;77(7):10751079 . 